\title{
Studying tunability of some NIR semiconductor lasers by external cavity setup
}

\section{Mohammad Amin Bani, Majid Nazeri, Hamed Abbasi}

Mohammad Amin Bani, Majid Nazeri, Hamed Abbasi, "Studying tunability of some NIR semiconductor lasers by external cavity setup," Proc. SPIE 10453, Third International Conference on Applications of Optics and Photonics, 1045310 (22 August 2017); doi: 10.1117/12.2272170

SPIE Event: Third International Conference on Applications of Optics and Photonics, 2017, Faro, Portugal 


\title{
Studying Tunability of Some NIR Semiconductor Lasers by External Cavity Setup
}

\author{
Mohammad Amin Bani ${ }^{\mathrm{a}}$, Majid Nazeri ${ }^{\mathrm{a} *}$, Hamed Abbasi ${ }^{\mathrm{b} *}$ \\ ${ }^{a}$ Faculty of Physics, University of Kashan, Kashan, Iran \\ ${ }^{\mathrm{b}}$ Biomedical Laser and Optics Group, Department of Biomedical Engineering, University of Basel, \\ Gewerbestrasse 14, CH-4123 Allschwil, Switzerland
}

\begin{abstract}
In this paper, tunability of three near-infrared semiconductor lasers which typically lase at $808 \mathrm{~nm}, 892 \mathrm{~nm}$ and $980 \mathrm{~nm}$ is studied. Both Littrow (wavelength dependent beam direction setup) and Littman (wavelength independent beam direction setup) configurations have been used. The wavelength and the power of the output beam of the laser have been measured. In all three cases, Littrow configuration shows a little better tuning range. In both Littrow and Littman configurations the wavelengths near the main wavelength of the laser had more power. The highest achieved power of these lasers in the Littrow setup was more than that of the Littman setup.
\end{abstract}

Keywords: External cavity, Littrow configuration, Littman configuration, semiconductor laser, near IR, tunability.

\section{INTRODUCTION}

In the middle of the $20^{\text {th }}$ century, finding the fact that a p-n junction can be used for generating coherence light led to a great revolution in laser industry and as a consequence in the related fields [1]. The smaller dimension and very lower weight compared to other types of lasers, as well as low power operation, desired efficiency, low price and good tunability range were the main advantage that made them a remarkable field of research. Along with the development of the sciences of physics, chemistry and electronics, the technology of this type of lasers has also improved. Developments in related fields such as deposition, power supplies and optical design led this type of lasers to become efficient and single mode lasers. This type of lasers can be used for various applications such as laser spectroscopy experiments, the telecommunications industry, a source for pumping other lasers, measuring and manipulating the quantum state of atoms, laser cooling and generation of terahertz radiation [2-9]. Nowadays, using lasers with varying wavelengths is an interesting field of research [10]; therefore, lasers will turn more applicable when their wavelengths can be tuned. There are a number of tuning mechanisms; one of them is using an external cavity as a feedback section. The principle of laser wavelength tuning by use of external feedback typically includes a wavelength-selective device positioned in the optical path of the laser beam that feeds a narrow part of the laser emission spectrum back into the laser cavity. There is another type of external-cavity laser that uses a resonator based on an optical fiber rather than on free-space optics. Tunable external cavity diode lasers (ECDLs) have been broadly developed. They have different applications in various fields of science. [11]. Tunable external cavity diode lasers (ECDLs) usually use a diffraction grating as the wavelength-selective element in the external resonator. In ECDLSs, the first-order of diffraction of the grating provides optical feedback to the laser diode chip. In this case a diode chip is required with a bandgap producing sufficient gain at the desired wavelength. The typical laser diode will have gain over several nanometers. ECDLs will often simultaneously support two or more closely-spaced longitudinal cavity modes with the total output power split between modes such that the effective power is substantially reduced. ECDLs have replaced complex and expensive traditional dye and Titanium Sapphire lasers as the workhorse laser of atomic physics labs $[8,9]$. The aim of this paper is to study tunability of three near-infrared semiconductor lasers with center wavelengths from $800 \mathrm{~nm}$ to $1000 \mathrm{~nm}$ in two different configurations (wavelength dependent and wavelength independent beam direction setup).

\footnotetext{
*m_nazeri@kashanu.ac.ir; phone +98 (0)31 559130 58; http://mnazeri.kashanu.ac.ir

*hamed.abbasi@unibas.ch; phone +41 (0)61 20754 61; http://dbe.unibas.ch/blog
}

Third International Conference on Applications of Optics and Photonics, edited by Manuel F. M. Costa, Proc. of SPIE Vol. 10453, 1045310 - @ 2017 SPIE · CCC code: 0277-786X/17/\$18 · doi: 10.1117/12.2272170 


\section{MATERIALS AND METHODS}

Due to the wide gain of semiconductor lasers, this type of lasers has a good ability for wavelength tuning. Many different configurations have been developed each having advantages and disadvantages. The most common configurations are the Littrow [12] and Littman [13] configurations while they are simple and applicable at the same time. In this paper, both Littrow and Littman configurations are used for tuning the lasers. A schematic diagram of Littrow configuration is shown in figure 1.

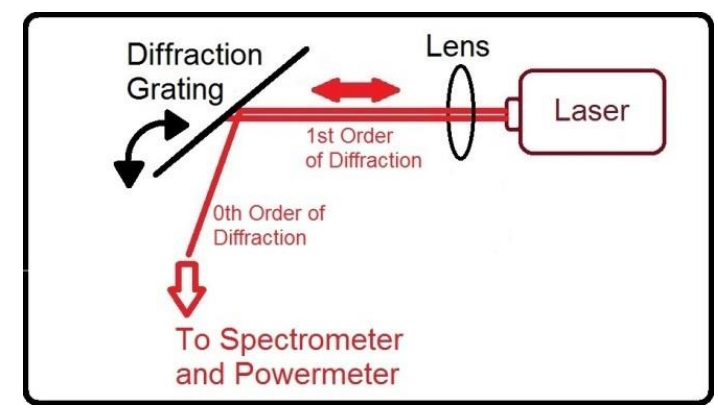

Figure 1. A schematic diagram of Littrow configuration.

As it has been shown in figure 1., the zeroth-order of the diffraction from the grating is used as the output beam of the tuned laser and the first-order of the diffraction from the grating is used as the feedback beam which is reflected back into the laser. In this setup, according to the angle between the laser and the diffraction grating, a specific wavelength is selected. Therefore, the output beam direction is wavelength dependent, leading to alignment problems when tuning the laser in some optical setups. If one needs wavelength independent beam direction, it can be achieved by adding an extra element, such as an intracavity beam splitter as an output coupler or a single plane mirror which is fixed relative to the tuning diffraction grating [14]. A schematic diagram of Littman configuration is shown in figure 2. The main difference between Littman and Littrow configurations is that the Littman configuration has an extra plane mirror compared to the Littrow configuration. In addition, the Littman configuration is more complex and requires a larger grating compared to the Littrow configuration [14].

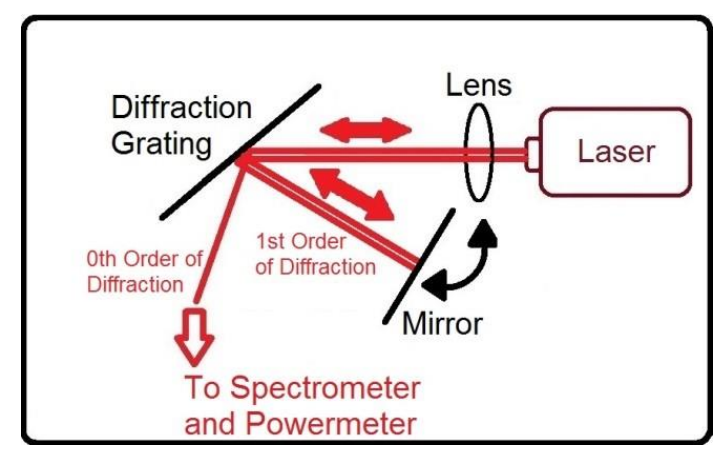

Figure 2. A schematic diagram of Littman configuration.

As it has been shown in figure 2., the Littman configuration uses a diffraction grating at near grazing incidence and an additional mirror. In this configuration, the first order of diffraction reflects back to the grating and the laser by the additional mirror. Therefore, in this setup, the wavelength is selected by the mirror angle, so that angle of the grating and the zeroth order of diffraction from the grating (the output beam) remain fixed during tuning the wavelength. One can use a temperature sensor and a thermoelectric cooler to control the temperature. A plane front surface mirror and a $1200 \mathrm{l} / \mathrm{mm}$ diffraction grating $(780 \mathrm{~nm}$ blaze) have been used in this research. The experiment has been carried out using three semiconductor lasers which typically lase at $806.3 \mathrm{~nm}(8.4 \mathrm{~mW}$, operating at $2.5 \mathrm{~V}$ and $80 \mathrm{~mA}$ current $), 888.9 \mathrm{~nm}(6.23$ $\mathrm{mW}$, operating at $2.4 \mathrm{~V}$ and $20 \mathrm{~mA}$ current $)$ and $980.6 \mathrm{~nm}(18.1 \mathrm{~mW}$, operating at $2.4 \mathrm{~V}$ and $50 \mathrm{~mA}$ current). The power of the laser was measured directly through the head of a digital optical power meter (Power Range: $50 \mathrm{nW}-50 \mathrm{~mW}$ ) and the wavelength of that was measured after passing the beam through the optical fiber ( 200 micron glass-core) of a compact CCD spectrometer (Spectral Range: 200 - $1000 \mathrm{~nm}$ ). 


\section{RESULT AND DISCUSSION}

The tuning range of 77 Angstrom was demonstrated in Littrow configuration for the laser with center wavelength of 806.3 $\mathrm{nm}$. Figure 3 shows the output power of the laser diode with center wavelength of $806.3 \mathrm{~nm}$ in different wavelengths in Littrow configuration.

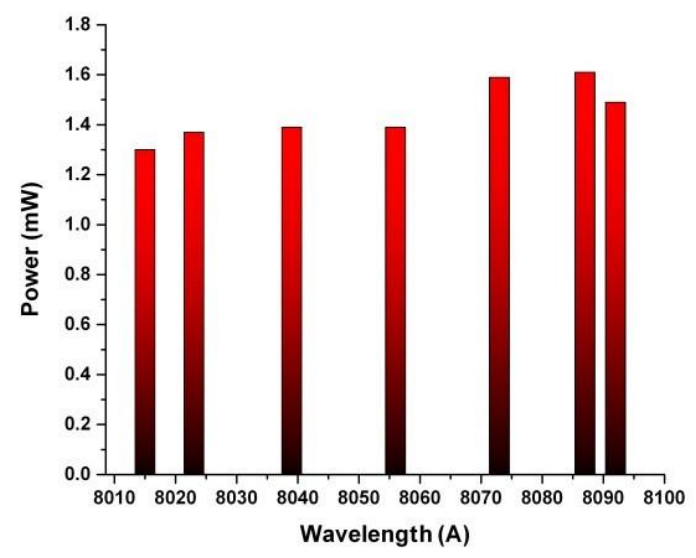

Figure 3. The output power of the laser diode with center wavelength of $806.3 \mathrm{~nm}$ in different wavelengths in Littrow configuration.

In figure 3, columns represent the power of the output beam of the laser in wavelengths of $801.5,802.3,8039,805.6$, 807.3, 808.7, and $809.2 \mathrm{~nm}$ respectively in $\mathrm{mW}$. The highest power achieved in this setup was $1.61 \mathrm{~mW}$ in $808.7 \mathrm{~nm}$.

In Littman configuration, the tuning range of 64 Angstrom was demonstrated for the laser diode with center wavelength of $806.3 \mathrm{~nm}$. Figure 4 shows the output power of the laser diode with center wavelength of $806.3 \mathrm{~nm}$ in different wavelengths in Littman configuration.

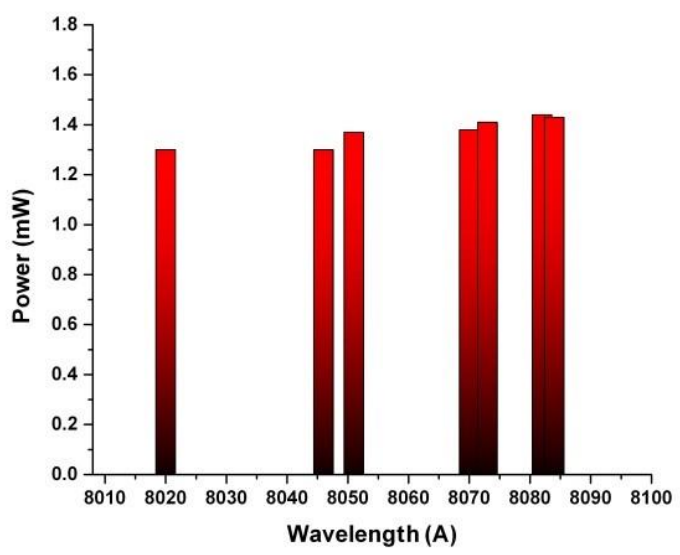

Figure 4. output power of the laser diode with center wavelength of $806.3 \mathrm{~nm}$ in different wavelengths in Littman configuration.

In figure 4, columns represent the power of the output beam of the laser in wavelengths of 802.0, 804.6, 805.1, 807.0, $807.3,808.2$, and $808.4 \mathrm{~nm}$ respectively in $\mathrm{mW}$. The highest power achieved in this setup was $1.44 \mathrm{~mW}$ in $808.2 \mathrm{~nm}$.

For the laser diode with center wavelength of $888.9 \mathrm{~nm}$, the tuning range of 154 Angstrom was demonstrated in Littrow configuration. Figure 5 shows the output power of the laser diode with center wavelength of $888.9 \mathrm{~nm}$ in different 
wavelengths in Littrow configuration. In both Littrow and Littman setups, when the wavelength of the laser approaches to the end of the tuning range, suddenly the output beam jumps to the main wavelength of the laser.

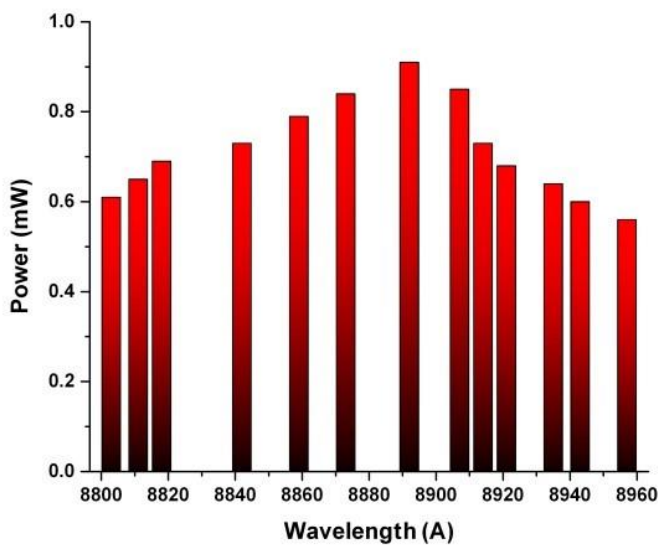

Figure 5. output power of the laser diode with center wavelength of $888.9 \mathrm{~nm}$ in different wavelengths in Littrow configuration.

In figure 5, columns represent the power of the output beam of the laser in wavelengths of $880.3,881.1,881.8,884.2$, $885.9,887.3,889.2,890.7,891.4,892.1,893.5,894.3$ and $895.7 \mathrm{~nm}$ respectively in $\mathrm{mW}$. As it is well visible in figure 5, the wavelengths near the main wavelength of the laser have more power. In this case, the highest power achieved in this setup was $0.91 \mathrm{~mW}$ in $889.2 \mathrm{~nm}$ which is very close to the main wavelength of the laser.

In Littman configuration of the laser diode with center wavelength of $888.9 \mathrm{~nm}$, the tuning range of 112 Angstrom was demonstrated. Figure 6 shows the output power of the laser diode with center wavelength of $888.9 \mathrm{~nm}$ in different wavelengths in Littman configuration.

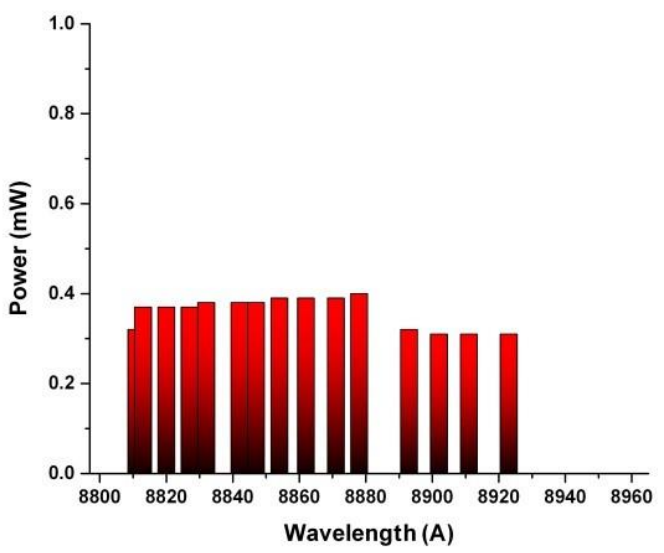

Figure 6. The output power of the laser diode with center wavelength of $888.9 \mathrm{~nm}$ in different wavelengths in Littman configuration.

In figure 6, columns represent the power of the output beam of the laser in wavelengths of 881.1, 881.3, 882.0, 882.7, $883.2,884.2,884.7,885.4,886.2,887.1,887.8,889.3,890.2,891.1$ and 892.3 respectively in $\mathrm{mW}$. The highest power achieved in this setup was $0.4 \mathrm{~mW}$ in 887.8 which is close to the main wavelength of the laser diode with center wavelength of $888.9 \mathrm{~nm}$. 
Finally, for the laser diode with center wavelength of $980.6 \mathrm{~nm}$, the tuning range of 130 Angstrom was demonstrated in Littrow configuration. Figure 7 shows the output power of the laser diode with center wavelength of $980.6 \mathrm{~nm}$ in different wavelengths in Littrow configuration.

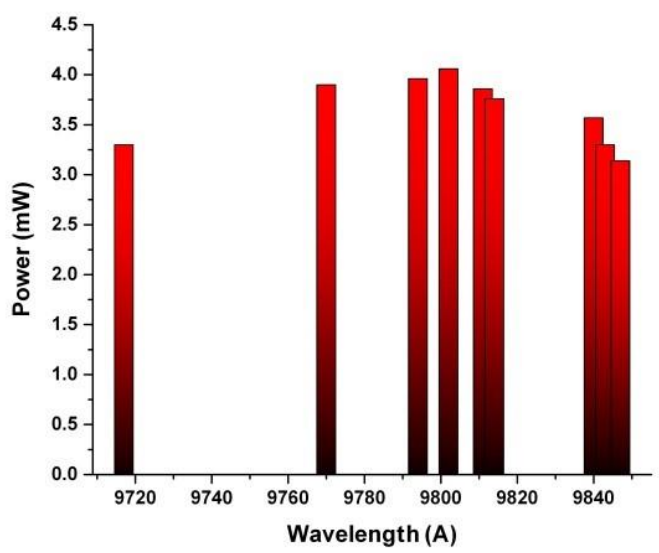

Figure 7. output power of the laser diode with center wavelength of $980.6 \mathrm{~nm}$ in different wavelengths in Littrow configuration.

In figure 7, columns represent the power of the output beam of the laser in wavelengths of 971.7, 977.0, 979.4, 980.2, 981.1, 981.4, 984.0,984.3, and 984.7 respectively in $\mathrm{mW}$. As it can be seen in figure 7, the highest power achieved in this setup was $4.06 \mathrm{~mW}$ in $980.2 \mathrm{~nm}$ which is very close to the center wavelength of the laser diode with center wavelength of $980.6 \mathrm{~nm}$.

In Littman configuration of the laser diode with center wavelength of $980.6 \mathrm{~nm}$ the tuning range of 55 Angstrom was demonstrated. Figure 8 shows the output power of the laser diode with center wavelength of $980.6 \mathrm{~nm}$ in different wavelengths in Littman configuration.

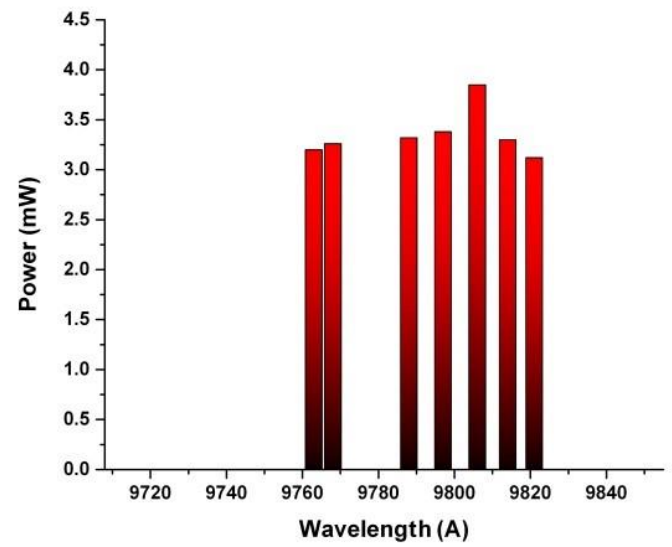

Figure 8. The output power of the laser diode with center wavelength of $980.6 \mathrm{~nm}$ in different wavelengths in Littman configuration.

In figure 8, columns represent the power of the output beam of the laser in wavelengths of 976.3, 976.8, 978.8, 979.7, 980.6, 981.4, and $982.1 \mathrm{~nm}$ respectively in $\mathrm{mW}$. As it can be seen in figure 8, the highest power achieved in this setup was $3.85 \mathrm{~mW}$ in $980.6 \mathrm{~nm}$ which is very close to the main wavelength of the laser diode with center wavelength of $980.6 \mathrm{~nm}$. 


\section{CONCLUSION}

The tunability of three semiconductor lasers with center wavelengths from 800 to $1000 \mathrm{~nm}$ in two different setups has been studied. Both Littrow (wavelength dependent beam direction setup) and Littman (wavelength independent beam direction setup) configurations have been employed in this experiment. The wavelength of the output beam of the laser has been measured by means of a grating spectrometer and the power of the output beam has been measured by means of a power meter. In all three cases, both configurations showed a good tuning range, but Littrow configuration shows a little better tuning range. The tuning ranges of 77 and 64 Angstrom for the laser diode with center wavelength of $806.3 \mathrm{~nm}$, the tuning range of 154 and 112 Angstrom for the laser diode with center wavelength of $888.9 \mathrm{~nm}$ and finally the tuning range of 130 and 55 Angstrom for the laser diode with center wavelength of $980.6 \mathrm{~nm}$ were demonstrated in Littrow and Littman configurations, respectively. In both Littrow and Littman setups, when the wavelength of the laser approaches to the end of the tuning range, suddenly the output beam jumped to the main wavelength of the laser. In both Littrow and Littman setups of all experiments the wavelengths near the main wavelength of the laser had more power. The highest achieved power of these lasers in the Littrow setup was more than that of the Littman setup which is probably related to the loss in reflection of an extra mirror and some misalignment problems.

\section{REFERENCES}

[1] Peyghambarian, N., Koch, S. W., Mysyrowicz, A., [Introduction to semiconductor optics], Prentice-Hall, Inc (1994)

[2] Duarte, F. J., [Tunable lasers handbook], Academic Press (1996)

[3] Duarte, F. J., [Tunable laser optics], $2^{\text {nd }}$ Ed. CRC Press (2015)

[4] Duarte, F. J., [Tunable laser applications], $2^{\text {nd }}$ Ed. CRC press (2009)

[5] Ye, C., Wei, T. K., [Tunable external cavity diode lasers], World Scientific (2004)

[6] Tani, M., Morikawa, O., Matsuura, S., Hangyo, M., "Generation of terahertz radiation by photomixing with dual- and multiple-mode lasers, Semicond. Sci. Tech. 20, 151-163 (2005).

[7] Bennetts, S., McDonald, G. D., Hardman, K. S., Debs, J. E., Kuhn, C. C., Close, J. D., Robins, N. P., "External cavity diode lasers with $5 \mathrm{kHz}$ linewidth and $200 \mathrm{~nm}$ tuning range at $1.55 \mu \mathrm{m}$ and methods for linewidth measurement", Opt. Exp. 22, 10642-10654, (2014)

[8] Hardman, K. S., Bennetts, S., Debs, J. E., Kuhn, C. C., McDonald, G. D., Robins, N., "Construction and Characterization of External Cavity Diode Lasers for Atomic Physics", J. Vis. Exp. 86, e51184 (2014).

[9] White, J. D., Scholten, R. E., "Compact diffraction grating laser wavemeter with sub-picometer accuracy and picowatt sensitivity using a webcam imaging sensor", Rev. Sci. Instrum. 83(11), 113104, (2012).

[10] Panahi, O., Nazeri, M., Tavassoli, S. H., "Design and construction of a tunable pulsed Ti: sapphire laser", J. Theor. Appl. Phys. 9(2), 99-103, (2015).

[11] Volodin, B. L., Dolgy, S. V., Melnik, E. D., Downs, E., Shaw, J., Ban, V. S., "Wavelength stabilization and spectrum narrowing of high-power multimode laser diodes and arrays by use of volume Bragg gratings", Opt. Lett. 29(16), 18911893 (2004).

[12] Hänsch, T. W., "Repetitively pulsed tunable dye laser for high resolution spectroscopy", App. Opt. 11, 895-898 (1972).

[13] Littman, M. G., Metcalf, H. J., "Spectrally narrow pulsed dye laser without beam expander", App. Opt. 17, 2224-2227 (1978).

[14] Hawthorn, C. J., Weber, K. P., "Scholten, R. E., Littrow configuration tunable external cavity diode laser with fixed direction output beam", Rev. Sci. Instrum. 72(12), 4477-4479 (2001). 